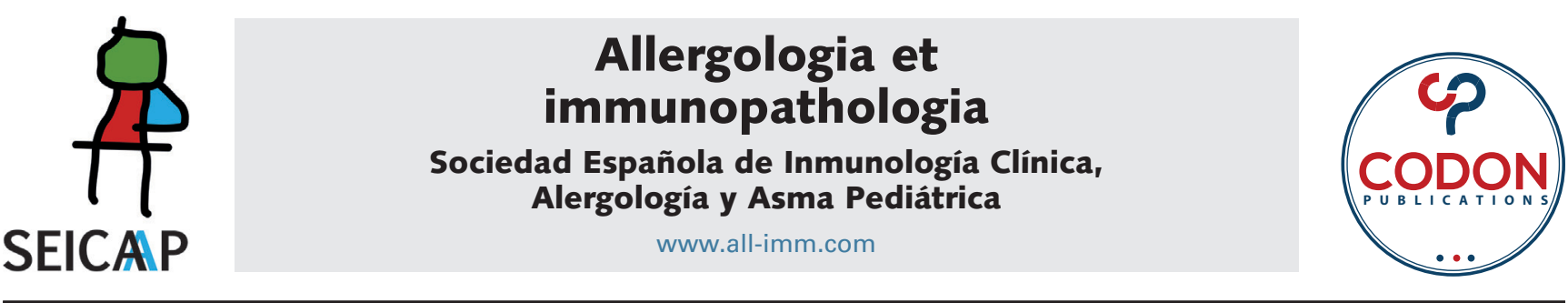

ORIGINAL ARTICLE

\title{
Efficacy of air purifier therapy for patients with allergic asthma
}

\author{
Luo Jia-ying ${ }^{\mathrm{a}}$, Ou Li-lia, Ma Jing ${ }^{\mathrm{b}}$, Lin Xin-yuan ${ }^{\mathrm{b}}$, Fan Li-min ${ }^{\mathrm{b}}$, Liu Hai-cheng ${ }^{\mathrm{b}}$, \\ Sun Bao-qing ${ }^{\text {** }}$
}

a State Key Laboratory of Respiratory Disease, National Clinical Centre for Respiratory Diseases, Guangzhou Institute of Respiratory Health, First Affiliated Hospital of Guangzhou Medical University, Guangzhou, China;

${ }^{b}$ Guangzhou Medical University, Guangzhou, China

Received 13 November 2020; Accepted 16 January 2021

Available online 1 September 2021

\section{KEYWORDS \\ air purifier; \\ allergic asthma; \\ fractional exhaled \\ nitric oxide; \\ house dust mite; \\ particulate matter}

\begin{abstract}
Objectives: This study aimed to evaluate the efficacy of air purifier therapy for patients with allergic asthma.

Methods: Thirty-eight subjects were categorized under two groups namely treatment group and control group. All subjects were under 18 years of age and they had been clinically diagnosed with allergic asthma. The treatment group used high efficiency particulate air (HEPA) purifiers for six consecutive months, and the control group did not use the air filters. Particulate matter (PM) data and dust samples (from bedding and a static point) were collected from the subjects' bedrooms before they started using the air purifiers and each month thereafter. Simultaneously, the subjects were asked to complete a questionnaire for the Asthma Control Test (ACT) or Childhood Asthma Control Test (C-ACT). Fractional exhaled nitric oxide (FENO) tests were performed at the start and end of the study. The concentrations of Der $\mathrm{p} 1$ and Der $\mathrm{f} 1$ were measured in the dust samples.

Results: (1) After utilizing the air purifier, the concentrations of house dust mite (HDM) allergens (Der p1+ Der f1) in the dust samples decreased. In addition, the PM indoor/outdoor values significantly decreased. (2) The ACT and C-ACT scores in the treatment group maintained a steady significant upward trend. (3) At the end of the study, the FENO levels in both groups were lower, although the differences were not significant.

Conclusions: It is witnessed that HEPA air purifiers can decrease indoor HDM allergen and PM levels and improve the quality of life for allergic asthma patients.
\end{abstract}

(c) 2021 Codon Publications. Published by Codon Publications.

*Corresponding author: Sun Bao-qing, State Key Laboratory of Respiratory Disease, National Clinical Centre for Respiratory Diseases, Guangzhou Institute of Respiratory Health, First Affiliated Hospital of Guangzhou Medical University, Guangzhou 510120, China. Email address: sunbaoqing@vip.163.com 


\section{Introduction}

Allergic asthma is the most common clinical chronic airway allergic disease. This disease leads to insomnia, daily fatigue, and decreased activity levels. Worldwide, 339 million people have asthma, ${ }^{1}$ and nearly $41 \%$ of asthma patients present allergic asthma. ${ }^{2}$ Epidemiological investigations have demonstrated that the prevalence of allergic asthma continues to increase. ${ }^{1}$ A large number of children have asthma. ${ }^{3}$ Therefore, this research mainly focused on children and teenagers under 18 years of age.

Allergic asthma is induced by exposure to environmental triggers, including air pollution and house dust mites (HDMs). ${ }^{1}$ Research by Falcon-Rodriguez $\mathrm{Cl}$ et al. ${ }^{4}$ showed that particulate matter (PM), the exposure to which increases oxidative stress and leads to asthma, is the main component of air pollution. Some studies have shown that the allergens that can trigger allergic asthma come not only from HDMs but also from other sources, including pollen, pet dander, pet hair, and insects. HDMs are the most prevalent allergen source in patients with asthma and/or rhinitis in China. ${ }^{5}$ Moreover, the HDM constitutes the main allergen affecting teenage asthma patients in Guangzhou, ${ }^{6}$ especially Der f1, which is the predominant mite allergen found in dust, with very high levels in bedding. ${ }^{7}$ Thus, decreasing the levels of PM and HDMs may be a feasible method of controlling asthma symptoms.

Some studies have shown that high efficiency particulate air (HEPA) air purifiers can trap air pollutants. ${ }^{8}$ However, whether avoiding exposure to environmental allergens would alleviate asthma patients' symptoms remains unclear. ${ }^{9}$ Therefore, this investigation aimed to determine the long-term efficacy of HEPA air purifiers for reducing $P M$ and HDM allergens and to evaluate the effect of air purifier use on subjects' asthma symptoms.

\section{Materials and Methods}

\section{Subject enrolment}

The subjects (the treatment and the control groups) were enrolled in this study at The First Affiliated Hospital of Guangzhou Medical University. The inclusion criteria were as follows: (1) Subjects were below 18 years of age. (2) Subjects had skin prick tests that were positive for Der $\mathrm{p} 1$ and Der $\mathrm{f} 1$ allergens. ImmunoCAP was performed to confirm the presence of Der p-specific IgE and Der $\mathrm{f}$-specific IgE in positive subjects (defined as $\geq 0.35 \mathrm{KU} / \mathrm{L}$ ). (3) According to the 2018 Global Strategy for Asthma Management and Prevention issued by the Global Initiative for Asthma (GINA), ${ }^{9}$ asthma can be classified according to severity. Subjects enrolled in this study were diagnosed with mild asthma (acute exacerbations could be alleviated by a short-term $\beta_{2}$ receptor agonist without the need for maintenance medication) and diagnosed based on GINA. Grouping was performed as 1:1 randomization. ${ }^{9}$ (4) During the study, the subjects were not allowed to change their place of residence. Subjects were required to adhere well to the instructions provided pertaining to the experiment (the treatment group was instructed to use the air purifier, complete the questionnaire, return for a follow-up visit, and allow the collection of samples; the control group was requested to complete the same steps, with an exception of using the air purifier). All subjects signed an informed consent form. The subjects completed the installation of the air purifier and started the experiment within 1 week after signing the informed consent form. The study was approved by the Ethics Committee of The First Affiliated Hospital of Guangzhou Medical University.

\section{Grouping and processing}

This study utilized a random number sequence to assign individuals to the treatment group and the control group; the treatment group used air purifiers, while the control group did not.

\section{Air purifier intervention in the treatment group}

This investigation provided a new HEPA air purifier (BA1030/1045, BRI air purifier, Xiamen, China) to every subject in the treatment group. The air purifier was placed in the bedroom of each subject, and they were asked to utilize the air purifier for six consecutive months (August 2016 to February 2017). Before the subjects used the air purifiers, they were taught by one of the researchers how to use the devices correctly. Researchers checked the air purifiers when they collected samples each month. The filter of each air purifier was cleaned after 3 months.

\section{Air purifier usage}

The air purifier was located at the head of each subject's bed. The air flow was carefully noted to prevent obstruction, and humidifiers were not permitted to be used at the same time. The doors and windows were kept closed when the air purifier was functioning. Moreover, the subjects were asked to use the air purifier every day and they were not allowed to clear the air filter themselves. The mean duration of daily HEPA air purifier use was $9.6 \pm 3.3 \mathrm{~h}$ and was fairly consistent during the course of the study.

\section{Return visit}

The researcher confirmed the air purifier placement and evaluated the subjects' correct usage of the air purifier on monthly basis. Incorrect usage was corrected. The control group did not receive any environmental interventions.

\section{Sample collection}

The researcher collected dust samples from the treatment and the control groups before the air purifiers were provided and on monthly basis after the use of the air purifiers was implemented. 


\section{Bedding dust sample collection}

Glass fiber filters and vacuum cleaners (Haier, ZW1401B) were the main tools used to collect dust samples from bedding. The glass fiber filter was set on the top of the collector and fixed there with a rubber band; the vacuum cleaner was used directly on the bedding. The duration of the sample collection was $15 \mathrm{~min}$.

\section{Static dust collection}

Through natural subsidence of dust particles, dust samples were collected in a static location by a glass fiber filter. Each glass fiber filter was placed in a $60-\mathrm{mm}$ culture dish. The dish was placed in a location with open exposure to the room and it could not be moved or covered during the month.

\section{PM data collection}

The researchers utilized a PM detector (DT-9881M, Huasheng Qi Xieco. Ltd., Shenzhen, China) to analyze indoor and outdoor $\mathrm{PM}^{2,5,10}$ concentrations. The machine was used for five-point collections (the middle and four corners of the room) indoors. Outdoor collections were performed in triplicate on the balcony or outside the window. The $\mathrm{PM}_{\text {indoor/outdoor }}$ ratio was recorded.

\section{Sample processing and extraction}

Every dust sample was weighed and recorded when it was collected. Then, the glass fiber filter was cut into pieces and placed on a $10-\mathrm{mL}$ syringe. Then, $1 \mathrm{~mL}$ [containing $1 \%$ BSA (bovine serum albumin) and $0.05 \%$ Tween-20] of phosphate-buffered saline/tween (PBST) was added, and the sample was extracted overnight at $4^{\circ} \mathrm{C}$ with shaking. The extracted mixture was removed from the injector and centrifuged at $4^{\circ} \mathrm{C}$ and $3000 \times \mathrm{g}$ for $30 \mathrm{~min}$. The supernatant was removed and stored at $-20^{\circ} \mathrm{C}$.

\section{HDM allergen content in dust}

This research utilized a double antibody sandwich enzyme-linked immunoassay (Indoor Biotechnologies, Charlottesville, VA, USA-ELISA) to test for two key HDM allergens, Der p1, and Der f1. According to the manufacturer's instructions, the concentrations of Der $\mathrm{p} 1$ and Der f1 were tested in each sample. The weight per gram of Der $\mathrm{p} 1$ and Der f1 were calculated from the concentrations of Der $\mathrm{p} 1$ and Der $\mathrm{f} 1$ and the weight of HDM.

\section{Evaluation of subjects}

The Asthma Control Test (ACT) and The Childhood Asthma Control Test (C-ACT) were used to evaluate the subjects' asthma control, while fractional exhaled nitric oxide (FENO) was used to evaluate the ability of the air purifiers to improve inflammation levels in the asthma patients.
$A C T$

ACT, which was designed by Nathan RA, ${ }^{10}$ is commonly used in asthma control research. It is an ideal method of evaluating asthma control. The questionnaire was completed by the subjects or their parents during each dust collection visit; ACT is only valid for adolescents aged 12 years or above.

\section{$C-A C T$}

C-ACT, designed by Liu $A H,{ }^{11}$ is often used in asthma control research. The questions are answered by the subjects' parents. The higher the score, the better the level of asthma control is. A score greater than 20 indicates that asthma is well controlled, while a score less than 20 indicates that asthma is not well controlled.

\section{FENO}

FENO is a marker of airway inflammation. Subjects underwent FENO tests (NIOX, Aerocrine, Sweden) $)^{12}$ at The First Affiliated Hospital of Guangzhou Medical University.

\section{Statistical analysis}

SPSS 19.0 (SPSS Inc, Chicago, IL, USA) was utilized to record and analyze data from the treatment and the control groups. Descriptive analysis was used to evaluate the subjects' basic characteristics (gender, age, slgE levels), the monthly HDM allergen concentrations, the PM levels, and the ACT and C-ACT scores. Normally distributed data are expressed as the mean $\pm \mathrm{SD}$, and non-normally distributed data are expressed as the median $\left[\mathrm{P}_{50}\left(\mathrm{P}_{25}, \mathrm{P}_{75}\right)\right]$. The Kolmogorov-Smirnov test was utilized to analyze the HDM allergen results. For normally distributed data, we used two independent samples for a non-parametric test analysis. According to the data distribution model, a two-tailed t-test or non-parametric test was used to compare the HDM allergen concentrations and FENO rates before and after using an air purifier. Moreover, repeated ANOVA was used to analyze the monthly HDM allergen concentrations, PM ratios, ACT scores, and C-ACT scores. $\mathrm{P}<0.05$ was considered statistically significant.

\section{RESULTS}

\section{Basic characteristics of the subjects}

Thirty-eight subjects were enrolled in this study. Both the treatment group and the control group had 19 subjects. These groups did not have any significant differences in basic characteristics $(P>0.05)$ (Table 1$)$.

\section{HDM concentration in the subjects' bedrooms}

The HDM concentration of bedding in the treatment group was $938 \mathrm{ng} / \mathrm{g}$, while that in the control group was much 
Table 1 Baseline characteristics of the subjects.

\begin{tabular}{lcc}
\hline & Treatment group & Control group \\
\hline Sex (male) & $10(52.6 \%)$ & $11(57.9 \%)$ \\
Age, years & $11 \pm 5$ & $10 \pm 3$ \\
Der p slgE (KU/L) & $62 \pm 36$ & $64 \pm 34$ \\
Der f slgE (KU/L) & $57 \pm 39$ & $81 \pm 32$ \\
FEV1 & $2.59 \pm 1.24$ & $1.65 \pm 8.00$ \\
FVC & $2.82 \pm 1.19$ & $2.05 \pm 0.83$ \\
PM2.5 & $1.04 \pm 0.56$ & $0.87 \pm 0.36$ \\
PM10 ${ }_{\text {indoor/outdoor }}$ & $1.09 \pm 0.54$ & $0.84 \pm 0.31$ \\
ACT & $19.7 \pm 2.7$ & $21.4 \pm 2.9$ \\
C-ACT & $21.7 \pm 3.9$ & $22.3 \pm 4.8$ \\
FENO & $52 \pm 36$ & $60 \pm 31$ \\
\hline
\end{tabular}

ACT, Asthma Control Test; C-ACT, Childhood Asthma Control Test; FVC, Forced Vital Capacity.

In addition to "Sex (male)," there were comparisons between the treatment group and the control group. $P>0.05$. Normal distributed data are expressed as the mean $\pm S D$, and non-normally distributed data are expressed as the quartile (P50 (P25, P75).
Table 2 The level of Der 1 allergen before treatment.

\begin{tabular}{llcc}
\hline & & Der p1 $(\mathrm{ng} / \mathrm{g})$ & Der f1 (ng/g) \\
\hline Bedding & Treatment group & $541 \pm 640^{\#}$ & $397 \pm 242^{\#}$ \\
& Control group & $48(12,305)^{\#}$ & $183 \pm 308^{\#}$ \\
Static & Treatment group & $165(157,239)^{*}$ & $60(59,61)^{*}$ \\
& Control group & $168 \pm 83^{*}$ & $45 \pm 35^{*}$ \\
\hline
\end{tabular}

${ }^{*} \mathrm{P}<0.05$, \# $\mathrm{P}>0.05$. Comparison between Der $\mathrm{p} 1$ and Der $\mathrm{f} 1$ in the same group.

lower, namely, $231 \mathrm{ng} / \mathrm{g}$. In addition, in the static samples, the HDM concentration in the treatment group was similar to that in the control group $(225 \mathrm{ng} / \mathrm{g}$ and $213 \mathrm{ng} / \mathrm{g}$, respectively).

There were no significant differences between the treatment group and the control group with respect to the Der p1 and Der $\mathrm{f} 1$ concentrations in the bedding and static samples $(P>0.05)$. The static dust samples from the treatment and control groups were mainly composed of Der p1. This finding demonstrated statistical significance. The concentration of Der $\mathrm{p} 1$ was greater than that
(A)
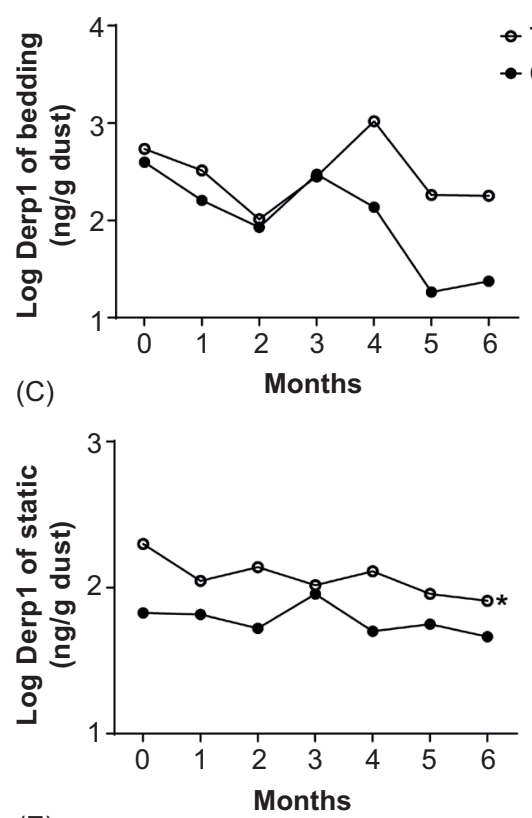

(E)

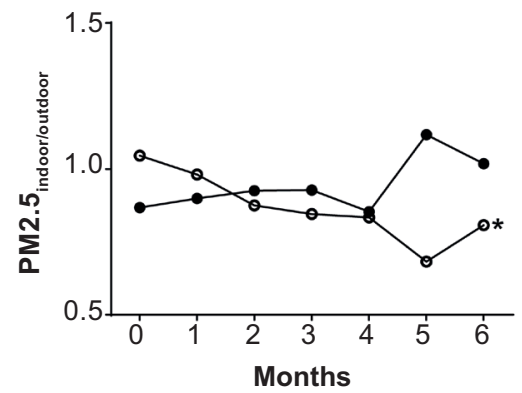

(B)
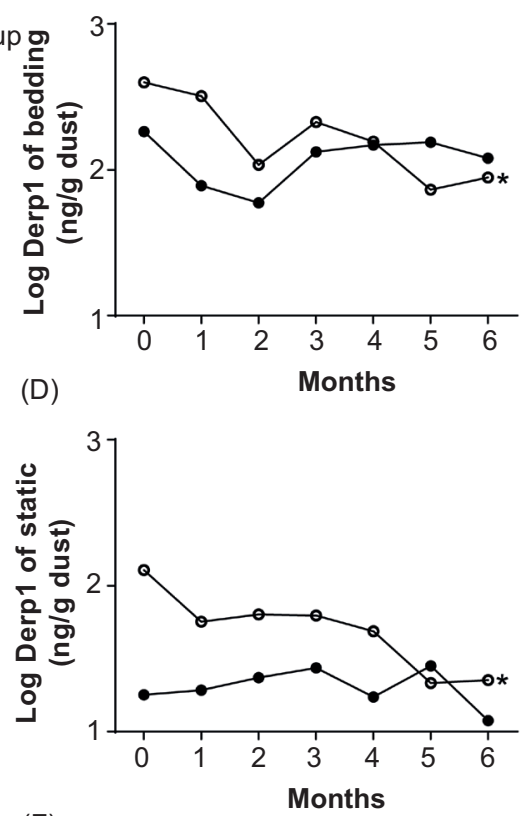

(F)

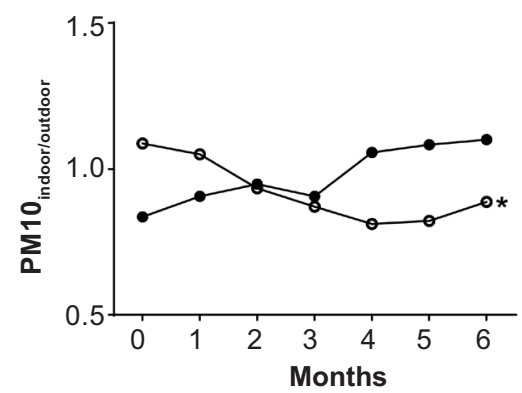

Figure 1 The concentration of HDMs in the house decreased, and the indoor/outdoor ratio of PM decreased significantly. (A-D) Variations in the log10 HDM allergen concentrations. (E-F) Variations in the $P M_{\text {indoor/outdoor }}$ ratio. *: $P<0.05$. Comparison of variations in the same group over time. HDMs, house dust mites. 
of Der $\mathrm{f1}$ in the bedding samples of the treatment group, while the concentration of Der $p 1$ was less than that of Der $\mathrm{f} 1$ in the bedding samples of the control group; however, this difference was not statistically significant $(P>$ 0.05) (Table 2).

\section{The influence of the air purifier on HDMs}

Repeated ANOVA showed that the Der f1 concentrations in the bedding and static dust samples and the concentration of Der $\mathrm{p} 1$ in the static dust samples changed significantly over time $(P<0.05)$. However, the increasing temporal trend of the Der p1 concentration in bedding dust samples did not reach statistical significance $(P>0.05)$ (Figure 1). Compared with the baseline values, the HDM allergen concentrations in bedding and static dust samples after using the air purifier were lower by $68.3 \%$ and $71.0 \%$, respectively. This finding was statistically significant. In the control group, the HDM allergen concentrations in bedding and static dust samples did not change consistently over time (Figure 1 (A, B, C, D)) and had not significantly decreased 6 months after the baseline $(P>0.05)$. The data points shown in the figures represent the mean of each group.

\section{Air purifier effect on PM}

After using an air purifier, the $\mathrm{PM}_{\text {indoor/outdoor }}$ ratio significantly decreased. $(P<0.05)$ Compared with the ratio after 5 months of using the air purifier, the $\mathrm{PM}_{\text {indoor/outdoor }}$ ratio recorded after the sixth month was slightly higher. However, the $\mathrm{PM}_{\text {indoor/outdoor }}$ ratio in the control group demonstrated a nonsignificant increasing trend $(P>0.05)$ (Figure 1E, F).

\section{Evaluation of symptom improvement ACT scores}

As shown in Figure 2, after using an air purifier, the ACT scores of the treatment group increased $(P<0.05)$ (Figure 2A), while those of the control group did not increase, indicating that the air purifier contributed to the control of asthma symptoms in the treatment group. Subjects in the treatment group experienced fewer asthma symptoms and instances of medication use at night compared with the baseline levels (Figure 3).

\section{C-ACT scores}

After utilizing the air purifier, the C-ACT scores of the treatment group increased $(P<0.05)$ (Figure $2 B)$. Moreover, the frequency of asthma symptoms at night also significantly decreased (Figure 4). However, the scores of the control group did not significantly increase (Figure 2B).

\section{Variation in FENO level}

After using the air purifier, the FENO values of the treatment group and the control group were $41 \pm 36$ and $52 \pm 36$, respectively, which were not significantly lower than the corresponding values at the baseline. $(P>0.05)$ (Figure 2C, D)
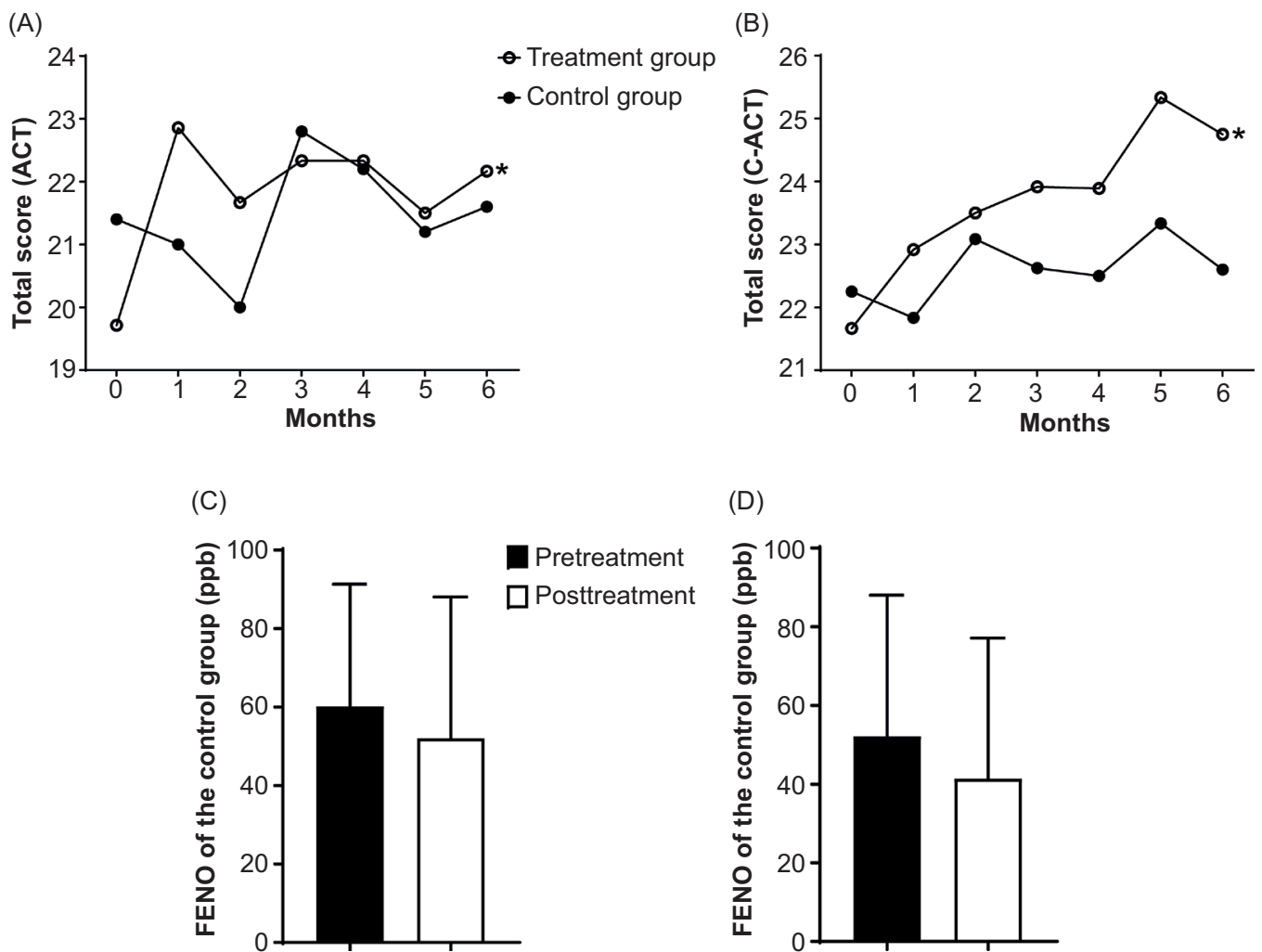

(D)

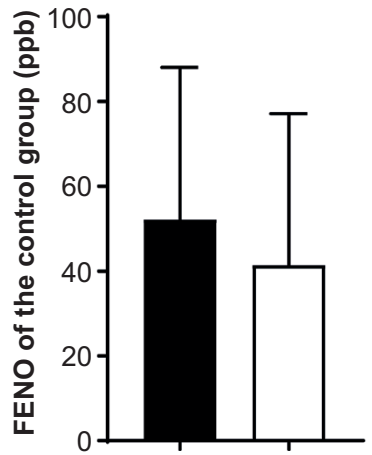

Figure 2 The use of air purifiers helped to control asthma symptoms in the treatment group. (A-B) Variations in the ACT/C-ACT total scores. ${ }^{*} \mathrm{P}<0.05$. Comparison of variations in the ACT/C-ACT total scores in the same group over time. (C-D) Variations in the FENO level. ACT, Asthma Control Test; C-ACT, Childhood Asthma Control Test. 
(A)

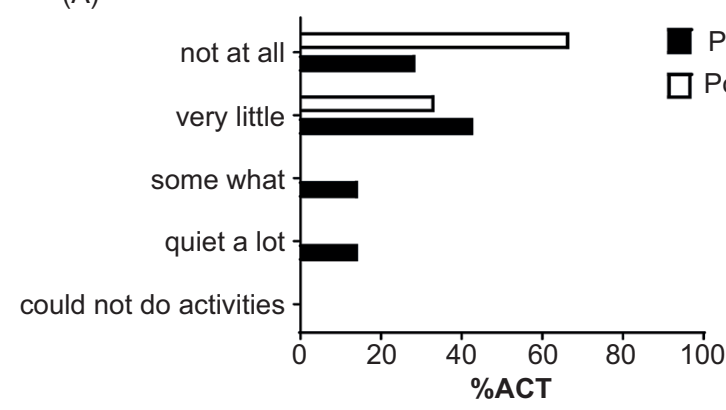

(C)

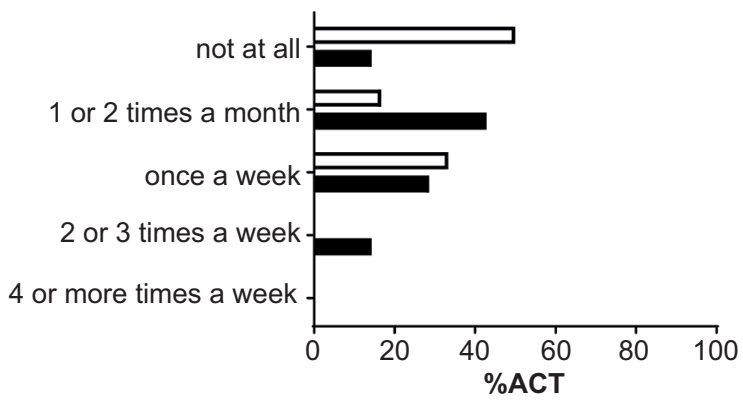

(B)

Pretreatment Posttreatment

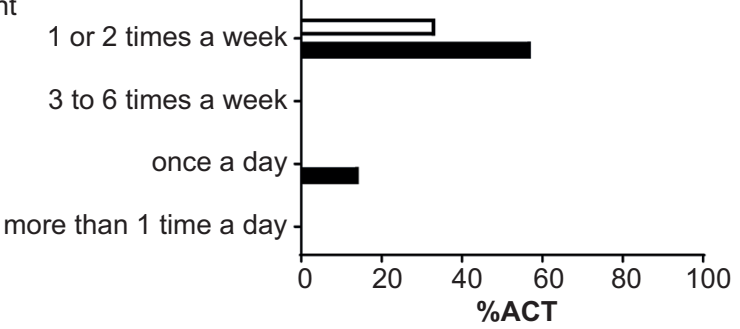

(D)

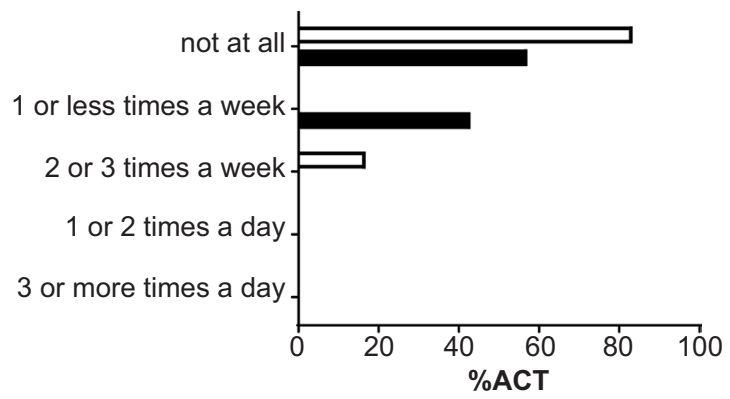

(E)

Figure 3 The ACT scores of the treatment group increased. (A) Percentages of the answers to the first question of the ACT questionnaire, "Does asthma restrict you in performing your usual daily activities?" (B) Percentages of the answers to the second question of the ACT questionnaire, "How often have you had shortness of breath?" (C) Percentages of the answers to the third question of the ACT questionnaire, "Do asthma symptoms wake you up at night or earlier than usual?" (D) Percentages of the answers to the fourth question of the ACT questionnaire, "Have you used your rescue inhaler or nebulizer medication?" (E) Percentages of the answers to the fifth question of the ACT questionnaire, "Rate your asthma control." ACT, Asthma Control Test.

\section{Discussion}

This research revealed that air purifiers were able to effectively improve asthma subjects' symptoms and facilitate asthma control by means of environmental intervention. This finding was determined by measuring the HDM and PM concentrations in asthma patients' bedrooms as well as the patients' symptoms and medication use.

After utilization of the air purifier, HDM allergen concentrations in the bedding and static dust samples significantly decreased. The decreases in HDM allergen concentrations in the static dust and bedding samples may be the results of the airflow generated by the air purifier and the filtering functionality.

Controlling asthma patients' symptoms is helpful in preventing asthma-related mortality and comorbidities. ${ }^{13}$
However, with the deteriorating quality of the environment, it is difficult to control the symptoms of patients with allergic asthma. ${ }^{14,15}$ Although environmental intervention has been used as an adjuvant therapeutic method, ${ }^{9}$ it is based on subjects' medication use, making it difficult to define its mechanism of action. Our research has demonstrated that air purifiers, representing a type of environmental intervention, can effectively decrease the concentrations of HDM allergens and PM. ${ }^{16}$ However, this experiment still did not measure asthma symptoms. Moreover, the observation time was short. Furthermore, in this study, all subjects had mild symptoms and did not need to take maintenance medications. Mild symptoms in asthma patients tend to be controlled by traditional Chinese medicine in China. ${ }^{17-19}$ If air purifiers represent an effective adjuvant therapeutic method, it will become 
(A)

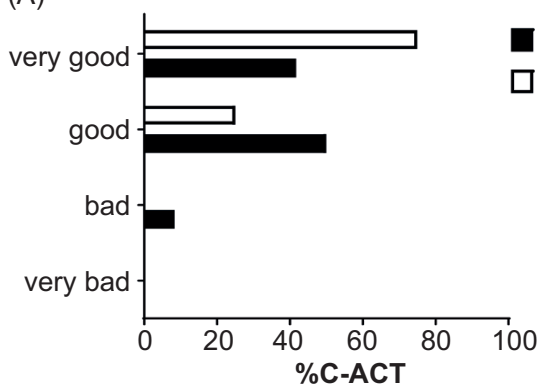

(C)

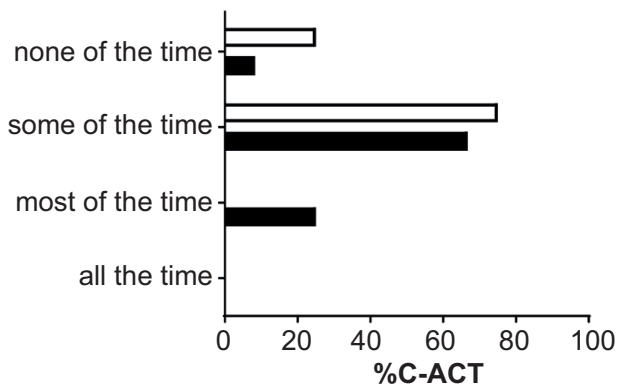

(E)

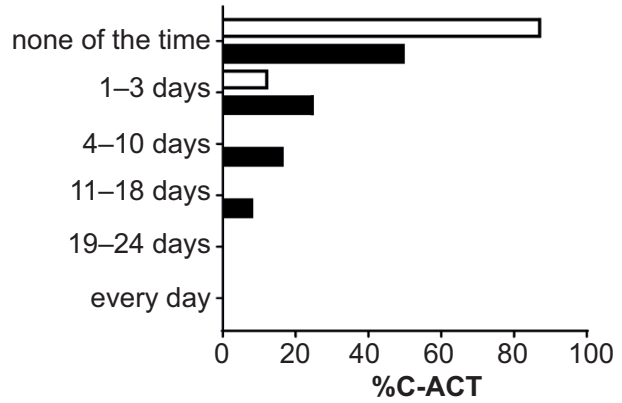

(B)

Pretreatment Posttreatment

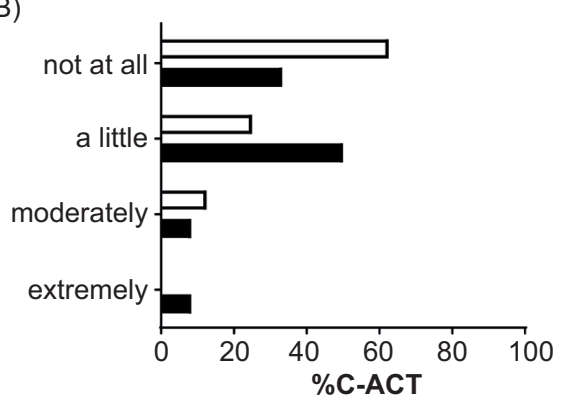

(D)

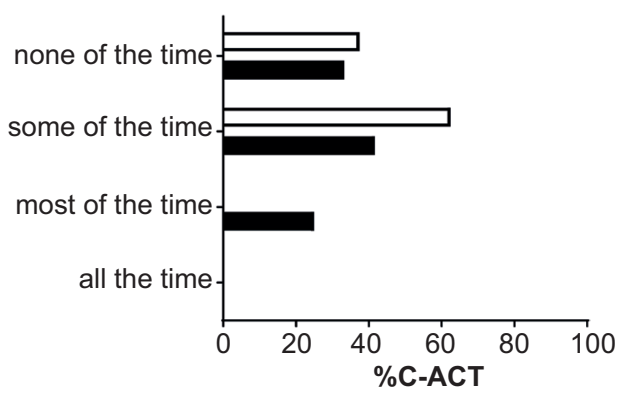

(F)

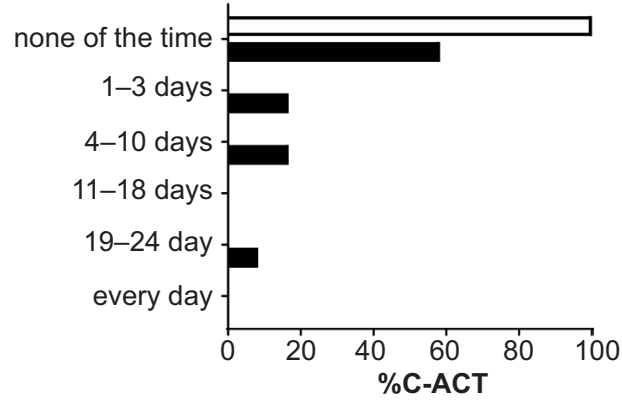

(G)

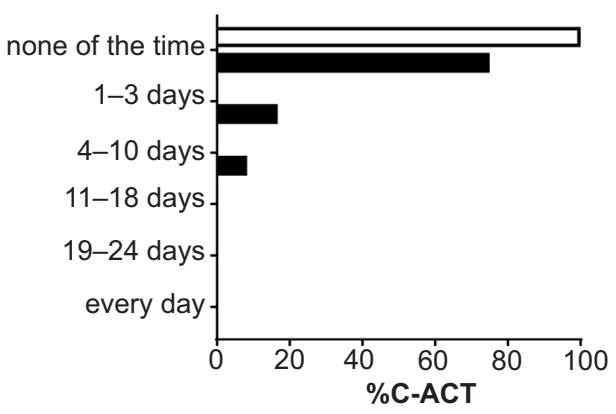

Figure 4 The C-ACT scores of the treatment group increased. (A) Percentages of the answers to the first question of the C-ACT questionnaire, "How is your asthma today?" (B) Percentages of the answers to the second question of the C-ACT questionnaire, "How much of a problem is your asthma when you run, exercise, or play sports?" (C) Percentages of the answers to the third question of the C-ACT questionnaire, "Do you cough because of your asthma?" (D) Percentages of the answers to the fourth question of the C-ACT questionnaire, "Do you wake up during the night because of your asthma?" (E) Percentages of the answers to the fifth question of the C-ACT questionnaire, "During the last 4 weeks, how many days did your child have any daytime asthma symptoms?" (F) Percentages of the answers to the sixth question of the C-ACT questionnaire, "During the last 4 weeks, how many days did your child wheeze during the day because of asthma?" (G) Percentages of the answers to the seventh question of the C-ACT questionnaire, "During the last 4 weeks, how many days did your child wake up during the night because of asthma?" C-ACT, Childhood Asthma Control Test. 
easier to control allergic asthma symptoms and prevent exacerbation of the disease. Although the FENO values in this study did not significantly change, there was a decreasing trend, which indicates that this method has a certain effect on asthma-related airway inflammation. According to the ACT/C-ACT scores, the subjects' frequency of awakening at night decreased with the use of the air purifier, which indicated that the subjects' nocturnal asthma symptoms were improved. Asthma symptoms at night are one of the main problems faced by asthmatic individuals; at night, mild asthma can be exacerbated. Moreover, asthma patients are more likely to develop obstructive sleep apnea than those without asthma. ${ }^{20}$ In addition, the ACT/C-ACT scores showed that subjects' daily limitations and medication frequency decreased after using the air purifier. The increased ACT/C-ACT scores demonstrated that the subjects' asthma symptoms were better controlled after using the air purifier. Xiang $L$ et al. ${ }^{21}$ demonstrated that exposure to Der $\mathrm{p} 1$ and Der $\mathrm{f} 1$ and ACT/C-ACT scores are negatively correlated. It is possible that the air purifier decreased the HDM allergen concentration in the bedroom, thereby improving the subjects' asthma symptoms. After the air purifier was used for 6 months, the PM2.5 and PM10 ratios significantly decreased. This find ing demonstrated that the HEPA air purifier could improve the air quality. Although this research utilized the ratio of indoor to outdoor PM concentrations to avoid the effect of variable outdoor PM concentrations, it cannot be ignored that the result may have been affected by external environmental factors. At the same time, this research also cannot exclude the effects of deviations in subjects' medication usage.

Paulin LM et al. ${ }^{22}$ indicated that the efficiency of purification will decrease after utilizing an air purifier for 3 months. However, it is still unclear whether the efficiency decreases because the filter element's efficiency decreases or because the filter screen's and filter element's efficiencies decrease at the same time; therefore, this study tried to explore this problem by cleaning the filter screen in the third month without replacing the filter element. The HDM allergen concentration in the bedding dust samples increased after 3 months, suggesting that the purification efficiency of the air purifier was decreasing. After the filter screen was cleaned, the bedding HDM allergen concentration decreased again. However, we did not find similar results in the other metrics used in this study. It is worth mentioning that the HDM allergen concentration in the bedding dust samples and the PM ratio increased after the sixth month of using the air purifier compared with the values after the fifth month. This finding may show that the air purifier's filter screen accumulated too much dust every 3 months, causing a decrease in the filter efficiency. The decrease in the filter efficiency may cause a decrease in the efficiency of the filter element and, consequently, a decrease in the overall purification efficiency of the device. Thus, preserving good conditions for the filter element by cleaning the filter screen every 3 months can help maintain the efficiency of the air purifier; moreover, air purification can be regarded as a feasible method of controlling asthma symptoms.

In general, this research revealed that HEPA air purifiers can effectively decrease indoor HDM allergen and PM levels and control allergic asthma symptoms. Moreover, the subjects' quality of life can be improved. However, this study did not use a placebo. Future investigations should include a placebo to further explore the effectiveness of air purifiers as a method of environmental intervention.

\section{Funding}

This study was supported by the Medical and Health Science and Technology Project in Guangzhou [grant number No. 20161A011066].

\section{Statement of Ethics}

This study has been approved by the ethics committee from the First Affiliated Hospital of Guangzhou Medical University (GYFYY-2016-73). The authors are accountable for all aspects of the work in ensuring that questions related to the accuracy or integrity of any part of the work are appropriately investigated and resolved.

\section{Acknowledgements}

We are deeply grateful to the patients for their excellent cooperation and to many other researchers for their assistance. Some of the preliminary results of the present study were presented by Luo JY as a poster at the ERS2016 meeting held in England on September 3, 2016. We are grateful for all the suggestions provided by experts. Moreover, we would like to express our deep appreciation to the editor and reviewers.

\section{Declaration of Interest}

The authors declare no direct, indirect, or personal interest.

\section{References}

1. The Global Asthma Network. The global asthma report 2018. Auckland, New Zealand: Global Asthma Network, 2018.

2. Romanet-Manent S, Charpin D, Magnan A, Lanteaume A, Vervloet D. Allergic vs nonallergic asthma: what makes the difference? Allergy. 2002;57:607-613. https://doi. org/10.1034/j.1398-9995.2002.23504.x

3. World Health Organization Asthma. [accessed on 16 October 2016]. https://www.who.int/news-room/fact-sheets/detail/ asthma

4. Falcon-Rodriguez $\mathrm{Cl}$, Osornio-Vargas AR, Sada-Ovalle I, Segura-Medina P. Aeroparticles, composition, and lung diseases. Front Immunol. 2016;7:3. https://doi.org/10.3389/ fimmu.2016.00003

5. Li J, Sun B, Huang Y, Lin X, Zhao D, Tan G, et al. A multicentre study assessing the prevalence of sensitizations in patients with asthma and/or rhinitis in China. Allergy. 2009;64:10831092. https://doi.org/10.1111/j.1398-9995.2009.01967.x

6. Li J, Wang H, Chen Y, Zheng J, Wong GW, Zhong N. House dust mite sensitization is the main risk factor for the increase in prevalence of wheeze in 13- to 14-year-old schoolchildren in Guangzhou city, China. Clin Exp Allergy. 2013;43:1171-1179. https://doi.org/10.1111/cea.12157 
7. Zhang C, Gjesing B, Lai X, Li J, Spangfort MD, Zhong N. Indoor allergen levels in Guangzhou city, southern China. Allergy. 2011;66:186-191. https://doi.org/10.1111/ j.1398-9995.2010.02465.x

8. Kajbafzadeh M, Brauer M, Karlen B, Carlsten C, van Eeden S, Allen RW. The impacts of traffic-related and wood smoke particulate matter on measures of cardiovascular health: a HEPA filter intervention study. Occup Environ Med. 2015;72:394400. https://doi.org/10.1136/oemed-2014-102696

9. Global Initiative for Asthma. Global strategy for asthma management and prevention, 2018. Available from: www.qinasthma.org.

10. Nathan RA, Sorkness CA, Kosinski M, Schatz M, Li JT, Marcus P, et al. Development of the asthma control test: a survey for assessing asthma control. J Allergy Clin Immunol. 2004;113:5965. https://doi.org/10.1016/j.jaci.2003.09.008

11. Liu AH, Zeiger R, Sorkness C, Mahr T, Ostrom N, Burgess S, et al. Development and cross-sectional validation of the childhood asthma control test. J Allergy Clin Immunol. 2007;119:817-825. https://doi.org/10.1016/j.jaci.2006.12.662

12. Dweik RA, Boggs PB, Erzurum SC, Irvin CG, Leigh MW, Lundberg JO, et al. An official ATS clinical practice guideline: interpretation of exhaled nitric oxide levels (FENO) for clinical applications. Am J Respir Crit Care Med. 2011;184:602-615. https://doi.org/10.1164/rccm.9120-11ST

13. Licari A, Brambilla I, Marseglia A, De Filippo M, Paganelli V, Marseglia GL. Difficult vs. severe asthma: definition and limits of asthma control in the pediatric population. From Pediatr. 2018;6:170. https://doi.org/10.3389/fped.2018.00170

14. Park S, Lee EH, Kho Y. The association of asthma, total IgE, and blood lead and cadmium levels. J Allergy Clin Immunol. 2016;138:1701-1703.e6. https://doi.org/10.1016/j. jaci.2016.04.030

15. Sierra-Heredia C, North M, Brook J, Daly C, Ellis AK, Henderson D, et al. Aeroallergens in Canada: distribution, public health impacts, and opportunities for prevention. Int J Environ Res Public Health. 2018;15:1577. https://doi. org/10.3390/ijerph15081577

16. Jia-Ying L, Zhao C, Jia-Jun G, Zi-Jun G, Xiao L, Bao-Qing S. Efficacy of air purifier therapy in allergic rhiniti. Asian Pac $\mathrm{J}$ Allergy Immunol. 2018;36:217-221. https://doi.org/10.12932/ AP-010717-0109

17. Lin PY, Chu CH, Chang FY, Huang YW, Tsai HJ, Yao TC. Trends and prescription patterns of traditional Chinese medicine use among subjects with allergic diseases: a nationwide population-based study. World Allergy Organ J. 2019;12:100001. https://doi.org/10.1016/j.waojou.2018.11.001

18. Wen MC, Wei CH, Hu ZQ, Srivastava K, Ko J, Xi ST, et al. Efficacy and tolerability of anti-asthma herbal medicine intervention in adult patients with moderate-severe allergic asthma. J Allergy Clin Immunol. 2005;116:517-524. https://doi. org/10.1016/j.jaci.2005.05.029

19. Chang TT, Huang $\mathrm{CC}$, Hsu CH. Clinical evaluation of the Chinese herbal medicine formula STA-1 in the treatment of allergic asthma. Phytother Res PTR. 2006;20:342-347. https:// doi.org/10.1002/ptr.1843

20. Wang Y, Liu K, Hu K, Yang J, Li Z, Nie M, et al. Impact of obstructive sleep apnea on severe asthma exacerbations. Sleep Med. 2016;26:1-5. https://doi.org/10.1016/j. sleep.2016.06.013

21. Xiang L, Fu Y, Wang J, Wang Q. [The correlation between the seasonal variation of house dust mite allergens exposure level in household and the level of asthma control in asthmatic children]. Zhonghua Er Ke Za Zhi. 2014;52:177-183.

22. Paulin LM, Diette GB, Scott M, McCormack MC, Matsui EC, Curtin-Brosnan J, et al. Home interventions are effective at decreasing indoor nitrogen dioxide concentrations. Indoor Air. 2014;24:416-424. https://doi.org/10.1111/ina.12085 